\title{
Dynamic Snap-Through of a Shallow Arch Under a Moving Point Load
}

\section{Jen-San Chen \\ Professor \\ e-mail: jschen@ccms.ntu.edu.tw \\ Jian-San Lin \\ Graduate Student}

Department of Mechanical Engineering, National Taiwan University, Taipei, Taiwan 10617
In this paper we study the dynamic behavior of a shallow arch under a point load $Q$ traveling at a constant speed. Emphasis is placed on finding whether snap-through buckling will occur. In the quasi-static case when the moving speed is almost zero, there exists a critical load $Q_{c r}$ in the sense that no static snap-through will occur as long as $Q$ is smaller than $Q_{c r}$. In the dynamic case when the point load travels with a nonzero speed, the critical load $Q_{c r}^{d}$ is, in general, smaller than the static one. When $Q$ is greater than $Q_{c r}^{d}$, there exists a finite speed zone within which the arch runs the risk of dynamic snap-through either while the point load is still on the arch or after the point load leaves the arch. The boundary of this dangerous speed zone can be determined by a more conservative criterion, which employs the concept of total energy and critical energy barrier, to guarantee the safe passage of the point load. This criterion requires the numerical integration of the equations of motion only up to the instant when the point load reaches the other end of the arch. [DOI: 10.1115/1.1804991]

\section{Introduction}

An arch subjected to lateral loads may become elastically unstable. If the initial height of the arch is of the same order as the span of the arch, the buckling deformation is nearly inextensional. On the other hand, an arch is termed shallow if the initial height is much smaller than the span. When the lateral load of a shallow arch reaches a critical value, the deformed shape may undergo a sudden jump called snap-through buckling. The buckling deformation of a shallow arch will be extensional rather than inextensional. Depending on how the lateral load is applied, the snapthrough buckling of a shallow arch can be divided into two categories, i.e., static buckling and dynamic buckling. In the case of static buckling, the lateral load is applied in a quasi-static manner. The first theoretical prediction on the static critical load was conducted by Timoshenko in 1935 [1], in which a pinned sinusoidal arch was subjected to a uniformly distributed load. Fung and Kaplan [2] extended the research by considering a flexibly supported shallow arch under various kinds of lateral loading, including a concentrated force acting at the midpoint of the arch. Gjelsvik and Bonder [3] presented a complete theoretical and experimental analysis on a clamped circular arch under a central concentrated load. Franciosi et al. [4] extended the conventional limit analysis to the collapse of arches under repeated loading. Schreyer and Masur [5] analyzed a clamped circular arch and demonstrated that the existence of a bifurcation of the equilibrium state is not an adequate condition for the use of the asymmetric buckling criterion. Lee and Murphy [6] considered the inelastic buckling of a clamped circular arch made of work-hardening material. Simitses [7] studied the effect of an elastic foundation on the critical loads of a sinusoidal arch under distributed loads. Roorda [8] conducted a series of experiments to study the effect of small imperfection on the buckling of elastic structures, including a laterally loaded circular arch.

In the case when the lateral load is applied suddenly instead of in a quasi-static manner, the phenomenon is dynamic and much more complicated. Generally speaking, the methodologies used in estimating dynamic critical loads of elastic structures can be classified into two groups [9]. The first approach is to study the total energy and the phase plane of the system. By this method, suffi-

Contributed by the Technical Committee on Vibration and Sound for publication in the JOURNAL OF VIBRATION AND ACOUSTICS. Manuscript received October 2002; final revision, March 2004. Associate Editor: Chin An Tan. cient conditions for dynamic stability may be established. The first theoretical prediction of dynamic buckling load was conducted by Hoff and Bruce in 1954 [10], in which they studied the stability of a sinusoidal arch under unit step loading and ideal impulsive loading. Hsu [11,12] and Hsu et al. [13] studied the effects of various parameters on the stability of a flexibly supported sinusoidal arch under impulsive and other types of time-varying loads. Xu et al. [14] considered a shallow arch elastically supported at both ends in the lateral direction and under impulsive loading. Lin and Chen $[15,16]$ studied the sufficient condition against dynamic snapthrough for a shallow arch under prescribed end motion. This approach provides a lower bound of the dynamic critical load.

The second approach is to solve the equations of motion numerically to obtain the system response and identify the critical load for specified system parameters. Humphreys [17] performed both numerical and experimental studies on the dynamic snapthrough of a circular arch under uniform impulsive loading. Lock [18] used both a numerical integration method and an infinitesimal stability analysis to predict the dynamic critical load of a sinusoidal arch under a step loading. Lo and Masur [19] presented a hybrid method for snap-through stability analysis, which incorporates an integral equation formulation in conjunction with a finite element method. Johnson and Mclvor [20] investigated numerically the effects of the spatial distribution of impulsive loads and damping [21] on the dynamic snap-through of a shallow arch. This approach provides a more accurate prediction of the critical load at the expense of a large amount of calculation.

In all this previous research, the lateral loading, either distributed or concentrated, is assumed to be fixed in space. In this paper we plan to study the dynamic stability of a shallow arch under a moving point force. The proposed problem is potentially important because shallow arches have been crucial elements in numerous structures for public transportation. For better understanding of the response and safety of these structures, it is necessary to study the behavior of an arch under high-speed moving loads. In the first part of this paper we study the quasi-static case when the moving speed of the point force is very small. Our analysis shows that there exists a static critical load in the sense that no static snap-through will occur as long as the point load is smaller than this critical load. The second part of the paper considers the case when the point load travels with a nonzero speed. Similar to the quasi-static case, there exists a dynamic critical load when the point load travels with a nonzero speed. The dynamic critical load 


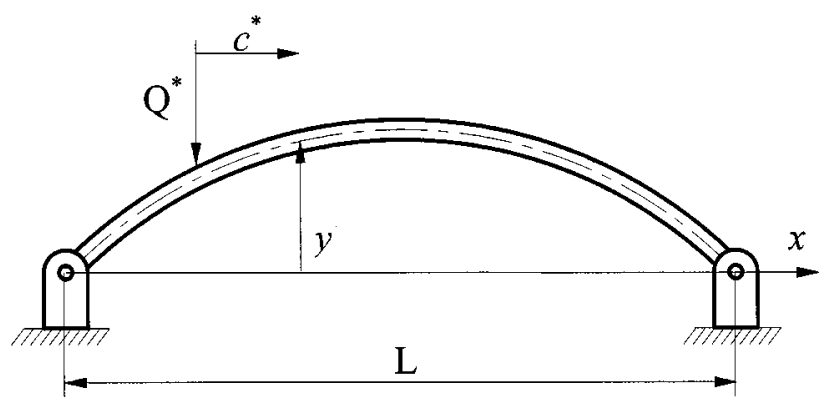

Fig. 1 Schematic diagram of a shallow arch under a moving point load

is, in general, smaller than the static one. When the point load is greater than the dynamic critical load, there exists a finite speed zone within which the arch runs the risk of dynamic snap-through. A simpler but more conservative criterion is then proposed to determine the boundary of this dangerous speed zone.

\section{Equations of Motion}

Figure 1 shows an elastic shallow arch with the two pinned ends being separated by a distance $L$. The initial shape of the unloaded arch is $y_{0}(x)$. The arch is subjected to a point force $Q^{*}$ traveling from $x=0$ to $x=L$ with a constant speed $c^{*}$. The equation of motion of the arch can be written as

$$
\rho A y_{, t t}=-E I\left(y-y_{0}\right)_{, x x x x}+p^{*} y_{, x x}-Q^{*} \delta\left(x-c^{*} t\right)
$$

The parameters $E, \rho, A$, and $I$ are Young's modulus, mass density, area, and moment of inertia of the cross section of the arch. $p^{*}$ is the induced axial force,

$$
p^{*}(t)=\frac{A E}{2 L} \int_{0}^{L}\left(y_{, x}^{2}-y_{0, x}^{2}\right) d x
$$

$\delta$ is the Dirac delta function. The boundary conditions for $y$ at $x$ $=0$ and $L$ are

$$
\begin{aligned}
y(0)-y_{0}(0) & =y_{, x x}(0)-y_{0, x x}(0)=y(L)-y_{0}(L) \\
& =y_{, x x}(L)-y_{0, x x}(L)=0
\end{aligned}
$$

Equations (1) and (2) can be nondimensionalized to the forms

$$
\begin{gathered}
u_{, \tau \tau}=-\left(u-u_{0}\right)_{, \xi \xi \xi \xi}+p u_{, \xi \xi}-\frac{\pi}{2} Q \delta(\xi-c \tau) \\
p=\frac{1}{2 \pi} \int_{0}^{\pi}\left(u_{, \xi}^{2}-u_{0, \xi}^{2}\right) d \xi
\end{gathered}
$$

where

$$
\begin{gathered}
u=\frac{y}{r}, \quad u_{0}=\frac{y_{0}}{r}, \quad \xi=\frac{\pi x}{L}, \quad \tau=\frac{\pi^{2} t}{L^{2}} \sqrt{\frac{E I}{A \rho}} \\
c=\frac{c^{*} L}{\pi} \sqrt{\frac{A \rho}{E I}}, \quad p=\frac{p^{*} L^{2}}{\pi^{2} E I}, \quad Q=\frac{2 Q^{*} L^{3}}{\pi^{5} E I r}
\end{gathered}
$$

$r$ is the radius of gyration of the cross section. $p=1$ corresponds to the Euler buckling load for a perfectly straight simply supported beam. $c=1$ corresponds to the speed of the flexural wave of the curved-beam with wave length $L$. It is noted that $Q$ is positive when the concentrated load points downward in Fig. 1.

In Eq. (1) we assume that the effect of the axial stress wave on the lateral vibration is negligible. This is true when the time spent by a compressive stress wave traveling back and forth over the arch for one run is smaller than the period for the arch to vibrate laterally. The ratio between the lateral period and the longitudinal period is proportional to the slenderness ratio $L / r$ of the arch.
Therefore, for an arch with $L / r=10$ for instance, the effect of the axial stress wave on the lateral vibration should be negligible as long as $c$ is smaller than 10 .

The initial shape of the arch before the lateral load is applied is assumed to be in the form

$$
u_{0}(\xi)=h \sin \xi
$$

$h$ is the rise parameter of the arch. It is assumed that the shape of the loaded arch can be expanded as

$$
u(\xi, \tau)=\lim _{N \rightarrow \infty} \sum_{n=1}^{N} \alpha_{n}(\tau) \sin n \xi
$$

After substituting Eqs. (7) and (8) into (4) and (5), we obtain the equations governing $\alpha_{n}$,

$$
\ddot{\alpha}_{n}=-n^{4} \alpha_{n}-n^{2} p \alpha_{n}-q_{n}, \quad n=1,2,3, \ldots
$$

where

$$
\begin{gathered}
p=\frac{1}{4} \sum_{k=1}^{\infty} k^{2} \alpha_{k}^{2}-\frac{h^{2}}{4} \\
q_{1}=Q \sin e-h \\
q_{n}=Q \sin n e, \quad n=2,3, \ldots \\
e(\tau)=c \tau
\end{gathered}
$$

The parameter $0<e(\tau)<\pi$ represents the position of the point load on the arch. The overhead dot in Eq. (9) represents differentiation with respect to $\tau$. The initial conditions for Eq. (9) are

$$
\begin{gathered}
\alpha_{1}(0)=h \\
\alpha_{n}(0)=0, \quad n=2,3, \ldots \\
\dot{\alpha}_{n}(0)=0, \quad n=1,2,3, \ldots
\end{gathered}
$$

\section{Equilibrium Configurations}

We first consider the case when the moving speed $c$ of the point load is small and the acceleration terms in Eq. (9) can be neglected. The equilibrium equations governing $\alpha_{n}$ can then be written as

$$
n^{4} \alpha_{n}+n^{2} p \alpha_{n}+q_{n}=0, \quad n=1,2,3, \ldots
$$

Equation (17) represents an infinite number of coupled nonlinear equations for the infinite number of coordinates $\alpha_{n}$. While it is, in general, impossible to solve for the infinite number of $\alpha_{n}$ simultaneously, it is possible to use a deduction method to derive the equation for $\alpha_{1}$, as demonstrated in the following.

$N=1$ : We first assume that the number of modes $N$ used in Eq. (8) is 1 . Then the solution $\alpha_{1}$ can be solved from the following cubic equation,

$$
f_{1}\left(\alpha_{1}\right)=\alpha_{1}+\frac{\alpha_{1}}{4}\left(\alpha_{1}^{2}-h^{2}\right)+q_{1}=0
$$

There are, at most, three one-mode equilibrium positions for $N$ $=1$.

$N=2:$ For the case when $N=2$ there are, at most, five equilibrium positions. After eliminating the axial thrust $p$ in Eq. (17) for $n=1$ and 2, we can derive the relation between $\alpha_{1}$ and $\alpha_{2}$ as

$$
\alpha_{2}=\frac{-\alpha_{1} q_{2}}{12\left[\alpha_{1}-\frac{q_{1}}{3}\right]}
$$

After substituting Eq. (19) into the first equation in (17), we obtain the equation for $\alpha_{1}$ as

$$
f_{2}\left(\alpha_{1}\right)=\left[\alpha_{1}-\frac{q_{1}}{3}\right]^{2} f_{1}\left(\alpha_{1}\right)+\frac{q_{2}^{2} \alpha_{1}^{3}}{144}=0
$$


(a)

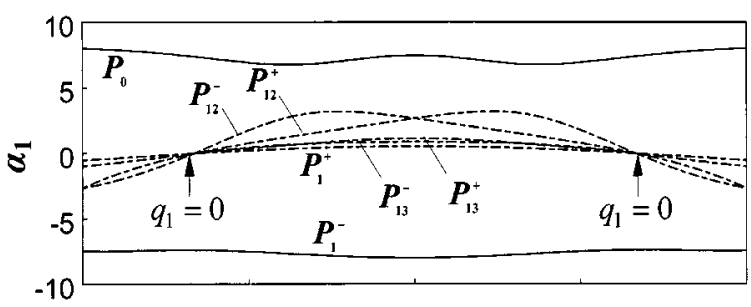

(b)

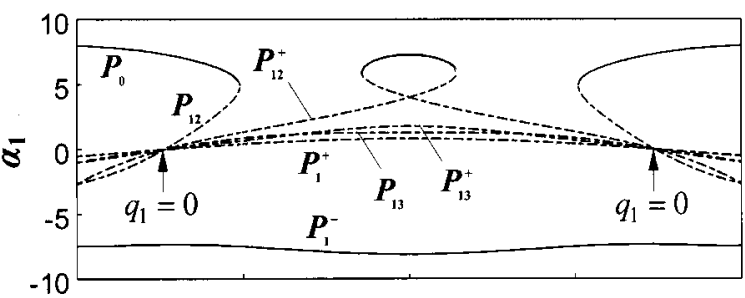

(c)

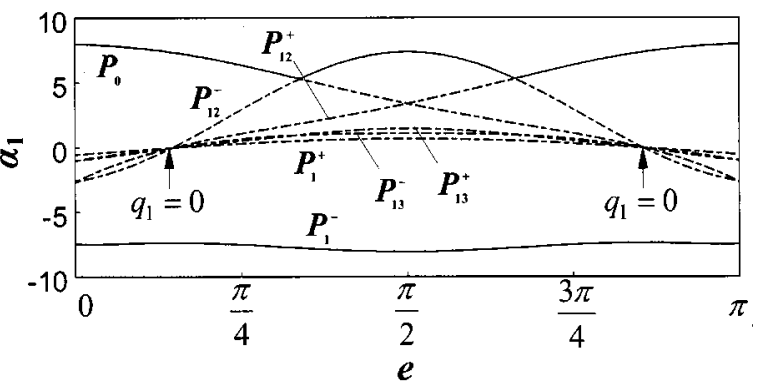

Fig. 2 Coordinate $\alpha_{1}$ as a function of load position $e$ for an arch with $h=8$ : (a) $Q=18$, (b) $Q=20$, and (c) $Q=18.16$

$N=k$ : There are, at most, $2 k+1$ equilibrium positions for the case when $N=k$. After eliminating $p$ in Eq. (17) for $n=1$ and $j$ we can derive the relation between $\alpha_{1}$ and $\alpha_{j}$ as

$$
\alpha_{j}=\frac{-\alpha_{1} q_{j}}{j^{2}\left(j^{2}-1\right)\left[\alpha_{1}-\frac{q_{1}}{\left(j^{2}-1\right)}\right]}, \quad j=2,3, \ldots, k
$$

After substituting Eq. (21) into the first equation in (17), we obtain the equation for $\alpha_{1}$,

$$
\begin{aligned}
f_{k}\left(\alpha_{1}\right)= & {\left[\alpha_{1}-\frac{q_{1}}{\left(k^{2}-1\right)}\right]^{2} f_{k-1}\left(\alpha_{1}\right)+\frac{q_{k}^{2} \alpha_{1}^{3}}{4 k^{2}\left(k^{2}-1\right)^{2}} \prod_{j=2}^{k-1}\left[\alpha_{1}\right.} \\
& \left.-\frac{q_{1}}{\left(j^{2}-1\right)}\right]^{2}=0
\end{aligned}
$$

$f_{k-1}\left(\alpha_{1}\right)=0$ is the equation for $\alpha_{1}$ when $N=k-1$.

\section{Convergence Test and Stability Analysis}

The first question in numerical calculation is how many modes should be included in the expansion (8). It is observed from Eq. (22) that the difference between using $k$ modes and $k-1$ modes in the expansion is the inclusion of the second term, which is inversely proportional to $k^{6}$. Therefore, we expect that the effects of the additional $k$ th harmonic in Eq. (8) on the original $2 k-1 \alpha_{1}$ 's when $k-1$ harmonics are used should be minimal for moderately large $k$.

In Fig. 2 we show the $\alpha_{1}$ as a function of load position $e$ for the case when $h=8$. Figures 2(a) -2 (c) are for $Q=18,20$, and 18.16, respectively. The number of modes $N$ used in the expansion is 4 . While there are, at most, nine equilibrium positions for $N=4$, only seven of them are real. In the case when $e=0$ (i.e., the arch is free from the point load), there are three one-mode solutions $\left(P_{0}, P_{1}^{+}, P_{1}^{-}\right)$involving only $\alpha_{1}$ and two pairs of two-mode solutions $\left(P_{12}^{ \pm}, P_{13}^{ \pm}\right)[15]$. It can be shown that for a free arch with $h>4$ only positions $P_{0}$ and $P_{1}$ are stable while all others are unstable. As the point load moves across the arch, these one and two-mode solutions will involve all the harmonic modes in expansion (8). However, we retain the names of the equilibrium positions when the point load moves across the arch. In Fig. 2(a) we observe that all the solution curves experience no bifurcation as the point load moves across the arch. Therefore, both $P_{0}$ and $P_{1}$ remain stable while all others remain unstable. We use solid and dashed lines to denote stable and unstable solutions. The stability properties of these equilibrium positions for a loaded arch can also be determined by the conventional energy method. As a consequence for $Q=18$ no snap-through will occur as the point load moves across the arch quasi-statically. However, it remains unknown yet whether snap-through will occur if the moving speed of the point load is no longer negligible. This will be a subject for later discussion.

In Fig. 2(b) for $Q=20$ we notice that as the point load moves across the arch the stable $P_{0}$ solution merges with the unstable $P_{12}^{-}$solution via a saddle-node bifurcation at $e=0.76$. As a consequence, the arch snaps from the $P_{0}$ position to the stable $P_{1}^{-}$ position at this bifurcation point. Three additional saddle-node bifurcation points are at $e=1.35,1.79$, and 2.38. As $Q$ decreases from 20 the saddle-node bifurcation points in Fig. 2(b) approach each other and eventually merge into transcritical bifurcation points when $Q=18.16$, as shown in Fig. 2(c). Therefore, $Q$ $=18.16$ is a critical load for $h=8$ in the sense that no snapthrough will occur when a point load smaller than this value moves across the arch quasi-statically.

It is noted that among the seven equilibrium positions in Fig. 2 , the solutions $P_{0}, P_{1}, P_{1}^{-}$, and $P_{12}^{ \pm}$are ultimately important. $P_{0}$ is the original configuration before snap-through buckling occurs. $P_{1}^{-}$is one of the two stable configurations. $P_{12}^{ \pm}$are important because snap-through buckling occurs when solution curve corresponding to $P_{0}$ merges with the solution curve corresponding to $P_{12}^{-}$via a saddle-node bifurcation, as shown in Figs. 2(b) and 2(c). Therefore, to capture the physical essence of the dynamic response we are interested in this paper and for the ease of analytical investigation, we propose to use only the first two harmonic modes in Eq. (8) in the following analysis. We compared the solution curves for $N=2$ to the results for $N=4$ in Fig. 2 and found that for $N=2$ and 4 the $\alpha_{1}$-curves of the five solutions $\left(P_{0}, P_{1}^{ \pm}, P_{12}^{ \pm}\right)$in which we are most interested are almost indistinguishable. More importantly, the saddle-node bifurcation points responsible for snap-through buckling are almost unchanged.

We observe in Fig. 2 that all solutions except $P_{0}$ and $P_{1}^{-}$meet at a point with $\alpha_{1}=0$ when $q_{1}=0$. The range of $e$ in the middle corresponds to $q_{1}>0$, while the ranges on both sides correspond to $q_{1}<0$. It is also observed that no snap-through will occur when $q_{1}<0$. In the range of $e$ where $q_{1}>0$ snap-through may [as in Fig. $2(b)]$ or may not [as in Fig. 2(a)] occur. These observations can actually be proved mathematically. The details of the proofs can be found in [22].

\section{Bifurcation Points}

As explained previously we can use only the first two modes in expansion (8), i.e., $N=2$, without losing any important dynamic characteristics in which we are interested. Equation (22) for $N$ $=2$ can be rewritten as

$$
\begin{gathered}
f_{2}\left(\alpha_{1}\right)=4\left(3 \alpha_{1}+h-Q \sin e\right)^{2}\left[4 \alpha_{1}+\left(\alpha_{1}^{2}-h^{2}\right) \alpha_{1}+4(Q \sin e\right. \\
-h)]+Q^{2} \alpha_{1}^{3} \sin ^{2} 2 e=0
\end{gathered}
$$

To locate the bifurcation point, we differentiate Eq. (23) with 

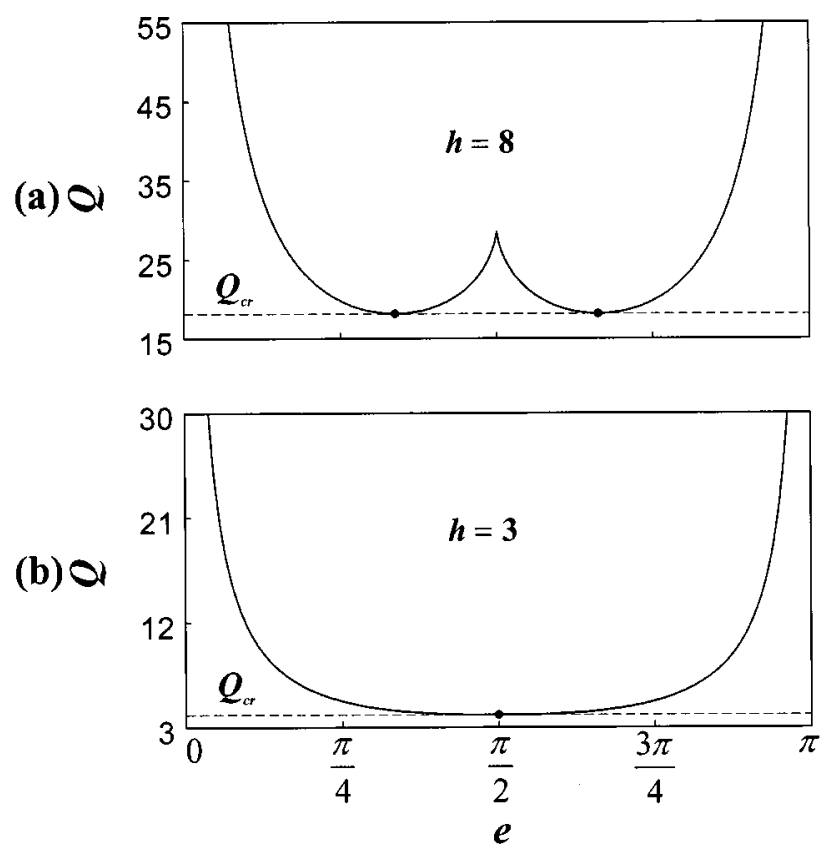

Fig. 3 Relation between load $Q$ and position $e$ when snapthrough occurs: (a) $h=8$ and (b) $h=3$

respect to $\alpha_{1}$. The resulting equation can be rearranged by using Eq. (23) in the following form:

$$
3 \alpha_{1}^{4}-\left(h^{2}+2\right)(Q \sin e-h) \alpha_{1}+6(Q \sin e-h)^{2}=0
$$

After specifying $e$ and $h$, we can eliminate $Q^{2}$ and $Q^{3}$ from Eqs. (23) and (24) to obtain a relation between $Q$ and $\alpha_{1}$. After substituting this relation back into Eq. (23), we can obtain a polynomial equation for $\alpha_{1}$. The highest order of this polynomial is 18 . The largest $\alpha_{1}$ represents the coordinate of the $P_{0}$ position when bifurcation occurs. The corresponding $Q$ is the load for snapthrough buckling. In this manner we can determine the relation between load $Q$ and position $e$ when snap-through occurs.

Figures $3(a)$ and $(b)$ show these relations for $h=8$ and 3, respectively. For the case when $h=8$ and $Q=20$, for instance, there are a total of four bifurcation points. Snap-through will occur as the load $Q=20$ moves from $e=0$ to the first bifurcation point $e$ $=0.76$. This result has already been reported in Fig. 2(b). When $Q$ is greater than 28.78 then there are only two bifurcation points. On the other hand, when $Q$ is smaller than 18.16 , then no snapthrough will occur when the point load moves across the arch quasi-statically. $Q=18.16$ can be considered as an upper limit for safe passage of the load across an arch with $h=8$, which is denoted by $Q_{c r}$ and signified by a horizontal dashed line in Fig. 3(a). There are two touching points between the dashed line and the bifurcation curve in Fig. 3(a). As $h$ decreases from 8, these two touching points will approach each other and eventually merge into one, as shown in Fig. 3(b) for the case when $h=3$. For this case there are, at most, two bifurcation points for a specified $Q$. The critical load $Q_{c r}$ for $h=3$ is 4.08 . Further calculation shows that for $h$ greater than 3.254 , there are two touching points as in Fig. 3(a). On the other hand, for $h$ smaller than 3.254, there will be only one touching point at the center as in Fig. 3(b). In other words, for an arch with $h<3.254$ the midpoint load is the worst case for static snap-through. On the other hand, for a higher arch with $h>3.254$, the worst case occurs when the point load is not at the center.

As demonstrated in Fig. 2(c) that the critical load $Q_{c r}$ corresponds to the situation when transcritical bifurcation occurs. The

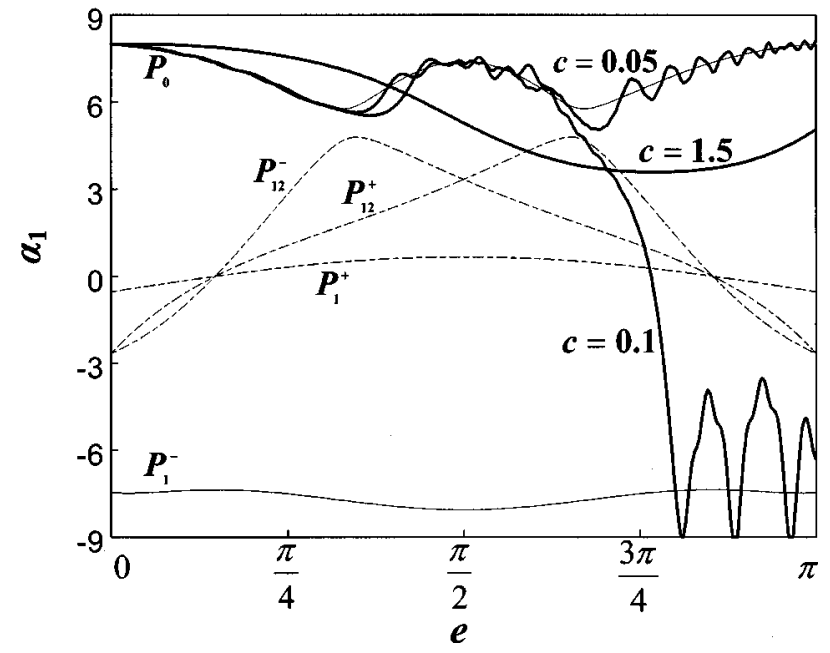

Fig. 4 The thick lines are the dynamic responses for an arch with $h=8, \mu=0.001$, and $Q=18$. The thin lines are the same quasi-static results as from Fig. 2(a).

additional condition for transcritical bifurcation is that the derivative of $f_{2}\left(\alpha_{1}\right)$ with respect to the control parameter $e$ vanishes [23], i.e.,

$$
\begin{gathered}
\left(3 \alpha_{1}+h-Q \sin e\right)\left[\alpha_{1}^{3}-\left(h^{2}+2\right) \alpha_{1}+6(Q \sin e-h)\right] \\
-Q \alpha_{1}^{3} \sin e \cos 2 e=0
\end{gathered}
$$

$Q_{c r}$ can be determined by substituting the relation between $Q$ and $e$ in Fig. 3 into Eq. (25) and checking if the equality sign in Eq. (25) is satisfied by a routine bisection approach. Further calculations show that $Q_{c r}$ increases with $h$.

\section{Dynamic Snap-Through}

The response of the arch will be different when the point load travels with a nonzero speed. The response history can be calculated by integrating Eq. (9) numerically with the initial conditions (14) $-(16)$. From the arguments cited in the preceding sections, we use only the first two equations with $n=1$ and 2 in Eq. (9) for simplicity. In the numerical simulation we also add damping terms $\mu \dot{\alpha}_{1}$ and $\mu \dot{\alpha}_{2}$ in these two equations of motion. It is noted that there is no technical difficulty in using more than two modes in expansion (8) for numerical simulation except that the calculation time will increase.

The quasi-static analysis in Fig. 2 shows that the arch with $h$ $=8$ will remain in the $P_{0}$ position as long as $Q$ is smaller than $Q_{c r}=18.16$. However, when the speed $c$ is increased from zero, dynamic snap-through may occur even when the load $Q$ is smaller than $Q_{c r}$. One of the thick lines in Fig. 4 shows the dynamic response of the arch with $h=8, Q=18, \mu=0.001$, and $c=0.1$. The arch snaps to the $P_{1}^{-}$position when the point load moves to the position $e=2.2$ and settles to $P_{1}^{-}$position thereafter. The equilibrium positions from quasi-static analysis $(c=0)$ are also shown in Fig. 4 with thin lines for comparison. On the other hand, when the speed $c$ is further increased to 1.5 , the arch does not have sufficient time to snap before the load reaches the other end and leaves the arch, as demonstrated by another thick line in Fig. 4. However, it must be emphasized that there still exists a possibility that the arch may continue to deform and snap to $P_{1}^{-}$position after the point load leaves the arch. Therefore, to determine whether the arch will snap or not, we have to continue the numerical simulation until the arch settles to one of the stable equilibrium positions. For the case with $c=1.5$ in Fig. 4 our numerical simulation shows that the arch will settle to $P_{0}$ position eventually. Furthermore, the arch never snaps to $P_{1}^{-}$at any instant before 


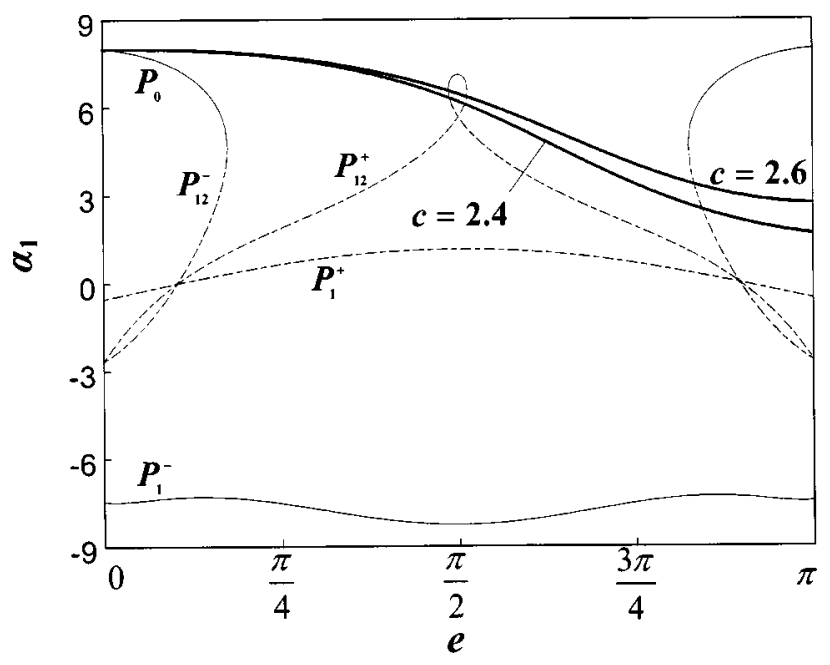

Fig. 5 The thick lines are the dynamic responses for an arch with $h=8, Q=25$, and $\mu=0.001$. The thin lines are the results from quasi-static analysis.

it settles to $P_{0}$. Therefore, in the case when $c=1.5$ no dynamic snap-through occurs when the point load is either still on the arch or after the point load leaves the arch.

It is noted in Fig. 4 that the static analysis does not predict the dynamic behavior for a small speed $c=0.1$. To demonstrate that the dynamic behavior indeed approaches the quasi-static result, we show the response for a smaller speed $c=0.05$. It can be seen that the curve for $c=0.05$ follows, with small oscillation, the quasi-static position $P_{0}$ between the two ends of the arch.

The analysis in Fig. 4 suggests that for an arch with $h=8$ and $Q=18$ there exist two critical speeds, denoted by ${c_{c r}^{-}}^{-}$and $c_{c r}^{+}$. The arch is safe from snap-through buckling as long as the moving speed $c$ is either smaller than $c_{c r}^{-}$or greater than $c_{c r}^{+}$. In other words, the speed range from $c_{c r}^{-}$to $c_{c r}^{+}$is the dangerous speed zone in the sense that the arch may snap dynamically. Obviously, $c_{c r}^{ \pm}$are functions of $Q$.

Figure 5 shows another case with $h=8$ and $Q=25$. The equilibrium positions from the quasi-static analysis are similar to those in Fig. 2(b) and are shown with thin lines. Apparently, for this case the critical speed $c_{c r}^{-}$is zero. On the other hand, when the moving speed is large enough, say, $c=2.6$, then the arch will not have enough time to snap before the load reaches the other end, as demonstrated by the thick line. Further calculation confirms that the arch settles to $P_{0}$ position after the point load leaves the arch. To demonstrate the situation that the arch may continue to deform and snap after the point load leaves the arch, we show the response history for a different speed $c=2.4$ in Fig. 5. Again the arch does not have enough time to snap before the load reaches the other end. However, further calculation shows that the arch will snap to $P_{1}^{-}$after the point load leaves the arch and settles to $P_{1}^{-}$eventually. The responses corresponding to $c=2.4$ and 2.6 after the point load leaves the arch are shown in Figs. 6(a) and $6(b)$, respectively. The damping coefficient $\mu$ used in these calculations is chosen to be 0.001 . The instant when the point load leaves the arch is signified by a black dot on the response curve.

\section{Boundary of Dangerous Speed Zone}

Figures 4-6 suggest that for an arch with two stable equilibrium positions when it is free of lateral loading, there may exist a dangerous speed zone if the point load is greater than a critical value $Q_{c r}^{d}$. Outside this dangerous speed zone the arch will not snap either while the point load is still on the arch or after the point load leaves the arch. The superscripted symbol $d$ indicates that this dynamic critical load is different from the critical load (a) $\overline{8}$

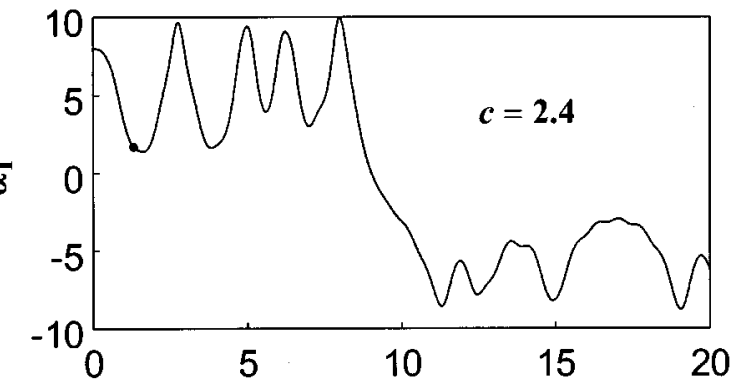

(b) $\bar{s}$

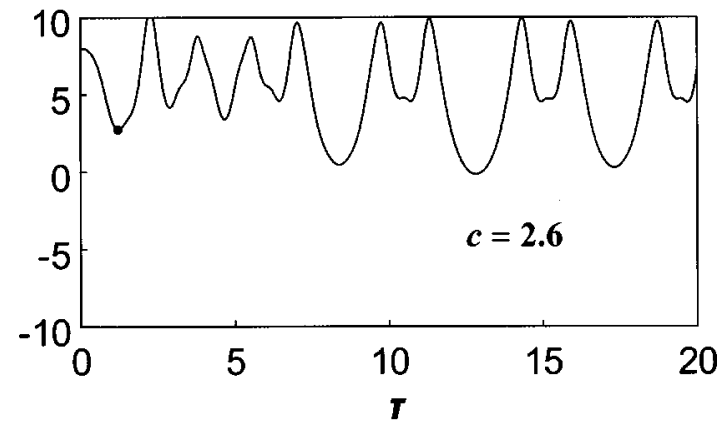

Fig. 6 Responses after the point load leaves the arch for the two speeds in Fig. 5: (a) $c=2.4$ and (b) $c=2.6$. The black dots signify the instant when the point load leaves the arch.

$Q_{c r}$ in Fig. 3, predicted from a quasi-static analysis. We are interested in comparing the critical loads from both the quasi-static and dynamic analyses. As explained in Figs. 4-6 to make certain whether the arch is safe, we have to observe the response history until the arch eventually settles to a stable equilibrium position. In general, it takes a long calculation time for the arch to settle. To simplify the calculation we notice that the arch will not snap if both of the following two conditions are satisfied: (i) the total energy $H$ gained by the arch at the instant when the point load reaches the other end is smaller than the critical energy barrier lying between the two distant stable equilibrium positions; (ii) the coordinate $\alpha_{1}$ remains greater than zero while the point load is still on the arch. It can be shown that for an arch with $4<h$ $<\sqrt{18}$, the critical energy barrier is the strain energy $U$ of position $P_{1}^{+}$. On the other hand, for an arch with $h>\sqrt{18}$, the critical energy barrier is the strain energy of position $P_{12}^{ \pm}$[15]. The two conditions can be stated mathematically as

$$
\begin{gathered}
H(e=\pi) \leqslant \operatorname{Min}\left[U\left(P_{1}^{+}\right), U\left(P_{12}^{ \pm}\right)\right] \\
\alpha_{1}(0<e<\pi)>0
\end{gathered}
$$

The total energy $H$ and the strain energy $U$ can be calculated as

$$
\begin{gathered}
H=2 p^{2}+\left(\alpha_{1}-h\right)^{2}+\sum_{n=2}^{\infty}\left[\dot{\alpha}_{n}^{2}+n^{4} \alpha_{n}^{2}\right] \\
U=2 p^{2}+\left(\alpha_{1}-h\right)^{2}+\sum_{n=2}^{\infty}\left[n^{4} \alpha_{n}^{2}\right]
\end{gathered}
$$

The physical total energy $H^{*}$ and strain energy $U^{*}$ are related to $H$ and $U$ by

$$
H^{*}=\frac{\pi^{4} E I^{2} H}{4 A L^{3}}, \quad U^{*}=\frac{\pi^{4} E I^{2} U}{4 A L^{3}}
$$

It is noted that these two conditions are sufficient in the sense that if they are satisfied, the arch will not snap from $P_{0}$ to $P_{1}^{-}$ either while the point load is still on the arch or after the point 


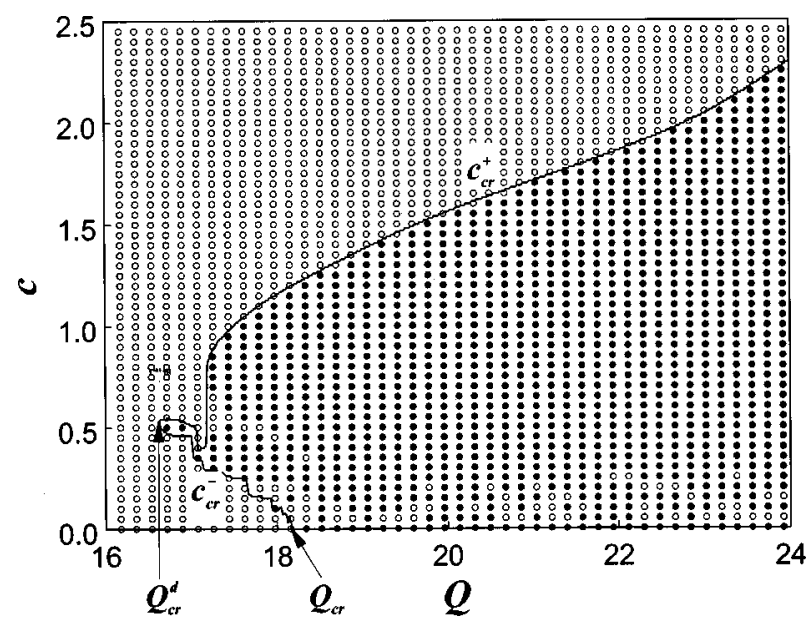

Fig. 7 Dangerous speed zone for an arch with $h=8$ and $\mu=0.001$. The solid circle indicates that the arch will snap dynamically before settling to a stable equilibrium position. The open circle indicates that the arch will never snap.

load leaves the arch. On the other hand, the arch may or may not snap if these two conditions are not satisfied. These conservative criteria allow us to determine the boundary of dangerous zone by integrating Eq. (9) up to the instant when the point load reaches the other end without going further thereafter.

In Fig. 7 we try to determine the dangerous speed zone in the $Q-c$ space for an arch with $h=8$. We divide the parameter space in Fig. 7 into $50 \times 50$ points. For each of these parameter points we examine conditions (26) and (27) and locate the point at which the equal sign of condition (26) is satisfied. The damping coefficient $\mu$ is chosen to be 0.001 . To verify the validity of these criteria we also continue the simulation until the arch settles to an equilibrium position. If the arch at any time snaps to position $P_{1}^{-}$, we put a solid circle at the parameter point. On the other hand, if the arch never snaps before it settles, then we put an open circle at the parameter point. It is observed that outside the dangerous speed zone only open circles are present. On the other hand, while most of the circles inside the dangerous zone are solid, there are a few open circles inside the dangerous speed zone. In the case when $c=0$, the critical load $Q_{c r}=18.16$ is predicted from quasi-static analysis. The dynamic critical load $Q_{c r}^{d}$ as defined above is 16.62 . In other words, the arch will not snap as long as the point load $Q$ is smaller than $Q_{c r}^{d}$ no matter what the moving speed is. In the case when $Q_{c r}^{d}<Q<Q_{c r}$ both $c_{c r}^{-}$and $c_{c r}^{+}$are nonzero. On the other hand when $Q_{c r}<Q, c_{c r}^{-}$is reduced to zero.

\section{Conclusions}

In this paper we study the dynamic behavior of a shallow arch under a point load $Q$ traveling at a constant speed $c$. Emphasis is placed on finding whether snap-through will occur if the arch possesses two stable equilibrium positions $P_{0}$ and $P_{1}^{-}$when it is free of lateral loading. The first part of the paper considers the quasi-static case when the moving speed is almost zero. Several conclusions can be summarized in this regard.

1. There exists a critical load $Q_{c r}$ in the sense that no static snap-through will occur as long as the load $Q$ is smaller than $Q_{c r}$.

2. In the case when $Q$ is greater than $Q_{c r}$, the solution curves of $P_{0}$ and $P_{12}^{-}$configurations will merge into a saddle-node bifurcation point. At the bifurcation point the arch will snap to the position $P_{1}^{-}$.

The second part of the paper considers the case when the point load travels with a nonzero speed. Some more conclusions can be summarized in this regard.
1. Similar to the quasi-static case there exists a dynamic critical load $Q_{c r}^{d}$ when the point load travels with a nonzero speed. No dynamic snap-through will occur as long as the point load $Q$ is smaller than $Q_{c r}^{d}$. The dynamic critical load $Q_{c r}^{d}$ is in general smaller than the static critical load $Q_{c r}$.

2. When the point load $Q$ is greater than $Q_{c r}^{d}$, there exists a finite speed zone between two critical speeds $c_{c r}^{-}$and $c_{c r}^{+}$, within which the arch runs the risk of dynamic snapthrough. In the case when $Q$ is greater than $Q_{c r}$, then $c_{c r}^{-}$is zero.

3. To determine the boundary of the dangerous speed zone we can use a more conservative criterion, which employs the concept of total energy and critical energy barrier, to guarantee the safe passage of the point load. This criterion requires the numerical integration of the equations of motion only up to the instant when the point load reaches the other end of the arch.

\section{Acknowledgment}

The results presented here were obtained in the course of research supported by a grant from the National Science Council of the Republic of China.

\section{References}

[1] Timoshenko, S. P., 1935, "Buckling of Flat Curved Bars and Slightly Curved Plates," ASME J. Appl. Mech., 2, pp. 17-20.

[2] Fung, Y. C., and Kaplan, A., 1952, "Buckling of Low Arches or Curved Beams of Small Curvature," NACA Technical Note 2840.

[3] Gjelsvik, A., and Bonder, S. R., 1962, "The Energy Criterion and Snap Buckling of Arches," J. Eng. Mech. Div., 88, pp. 87-134.

[4] Franciosi, V., Augusti, G., and Sparacio, R., 1964, "Collapse of Arches Under Repeated Loading," J. Struct. Div. ASCE, 90, pp. 165-201.

[5] Schreyer, H. L., and Masur, E. F., 1966, "Buckling of Shallow Arches," J. Eng. Mech. Div., 92, pp. 1-19.

[6] Lee, H. N., and Murphy, L. M., 1968, "Inelastic Buckling of Shallow Arches," J. Eng. Mech. Div., 94, pp. 225-239.

[7] Simitses, G. J., 1973, "Snapping of Low Pinned Arches on an Elastic Foundation," ASME J. Appl. Mech., 40, pp. 741-744.

[8] Roorda, J., 1965, "Stability of Structures With Small Imperfections," J. Eng. Mech. Div., 91, pp. 87-106.

[9] Simitses, G. J., 1990, Dynamic Stability of Suddenly Loaded Structures, Springer-Verlag, New York.

[10] Hoff, N. J., and Bruce, V. G., 1954, "Dynamic Analysis of the Buckling of Laterally Loaded Flat Arches," J. Math. Phys., 32, pp. 276-288.

[11] Hsu, C. S., 1967, "The Effects of Various Parameters on the Dynamic Stability of a Shallow Arch," ASME J. Appl. Mech., 34, pp. 349-358.

[12] Hsu, C. S., 1968, "Stability of Shallow Arches Against Snap-Through Under Timewise Step Loads," ASME J. Appl. Mech., 35, pp. 31-39.

[13] Hsu, C. S., Kuo, C. T., and Lee, S. S., 1968, "On the Final States of Shallow Arches on Elastic Foundations Subjected to Dynamical Loads," ASME J. Appl. Mech., 35, pp. 713-723.

[14] Xu, J.-X., Huang, H., Zhang, P.-Z., and Zhou, J.-Q., 2002, "Dynamic Stability of Shallow Arch With Elastic Supports-Application in the Dynamic Stability Analysis of Inner Winding of Transformer During Short Circuit,” Int. J. NonLinear Mech., 37, pp. 909-920.

[15] Lin, J.-S., and Chen, J.-S., 2003, "Dynamic Snap-Through of a Laterally Loaded Arch Under Prescribed End Motion," Int. J. Solids Struct., 40, pp. 4769-4787.

[16] Chen, J.-S., and Lin, J.-S., 2004, "Effects of Prescribed End Motion on the Dynamic Stability of a Shallow Arch on an Elastic Foundation," J. Eng. Mech. Div., 130, pp. 359-362.

[17] Humphreys, J. S., 1966, "On Dynamic Snap Buckling of Shallow Arches,' AIAA J., 4, pp. 878-886.

[18] Lock, M. H., 1966, "The Snapping of a Shallow Sinusoidal Arch Under a Step Pressure Load," AIAA J., 4, pp. 1249-1256.

[19] Lo, D. L. C., and Masur, E. F., 1976, "Dynamic Buckling of Shallow Arches,' J. Eng. Mech. Div., 102, pp. 901-917.

[20] Johnson, E. R., and Mclvor, I. K., 1978, "The Effect of Spatial Distribution on Dynamic Snap-Through," ASME J. Appl. Mech., 45, pp. 612-618.

[21] Johnson, E. R., 1980, "The Effect of Damping on Dynamic Snap-Through," ASME J. Appl. Mech., 47, pp. 601-606.

[22] Lin, J.-S., 2002, Dynamic Stability of a Shallow Arch Under Prescribed End Motion, Master Thesis, Department of Mechanical Engineering, National Taiwan University, Taipei, Taiwan.

[23] Nayfeh, A. H., and Balachandran, B., 1995, Applied Nonlinear Dynamics, Wiley, New York. 